\section{An improved mRFP1 adds red to bimolecular fluorescence complementation}

\author{
Guido Jach ${ }^{1}$, Martina Pesch ${ }^{2}$, Klaus Richter ${ }^{1}$, \\ Sabine Frings ${ }^{1}$ \& Joachim F Uhrig ${ }^{1,2}$
}

\begin{abstract}
Protein-protein interactions are fundamental to virtually every aspect of cellular functions. Blue, green and yellow bimolecular fluorescence complementation (BiFC) systems based on GFP and its variants allow the investigation of protein-protein interactions in vivo. We have developed the first red BiFC system based on an improved monomeric red fluorescent protein (mRFP1-Q66T), expanding the range of possible applications for BiFC.
\end{abstract}

With interactome data available for several model organisms, a challenging next step in post-genomic research is to analyze protein complex formation in vivo. The recently developed BiFC assay is a comparably fast and simple noninvasive technology to study protein interactions inside living cells. BiFC is based on the reconstitution of the yellow fluorescent protein (YFP) from two nonfluorescent fragments when they are brought into close proximity by a physical interaction between proteins fused to each fragment ${ }^{1}$. This approach has proven to be robust and versatile, and multicolor versions using spectral variants of GFP further increase the potential of this technology ${ }^{2}$. The color spectrum available for BiFC, however, has been limited to blue, green and yellow.

The red fluorescent protein from Discosoma sp. (DsRED) and its variants are established intracellular reporter proteins, but the characteristics of most DsRED variants, particularly their obligate tetramerization, impede their application in $\mathrm{BiFC}^{3,4}$. An extensively mutated monomeric DsRED variant (mRFP1) has been generated ${ }^{5}$, but substantially altered spectra, poor brightness and low photostability limit its usefulness as a reporter protein.

We have identified improved mRFP1 mutants, which allowed us to establish a red fluorescent reporter system for detection of protein interactions in vivo. We used site-directed mutagenesis to generate mRFP1 variants with a randomized first position of the fluorophore. Screening of 5,000 colonies resulted in the identification of 50 clones exhibiting strong red fluorescence representing three different mutations (Q66C, Q66S and Q66T) with frequencies of $5 \%, 43 \%$ and $52 \%$, respectively.
In vitro analyses of purified recombinant proteins mRFP1-Q66C, mRFP1-Q66S and mRFP1-Q66T revealed substantially altered spectral properties, whereas controls (wild-type DsRED and mRFP1) were in accordance with published data ${ }^{5}$ (Fig. 1a,b). Compared to mRFP1, the excitation and emission peaks of these new variants are shifted toward shorter wavelengths and resemble those of DsRED (Table 1). Absorption spectra of mRFP1, mRFP1Q66C and mRFP1-Q66S showed additional absorption peaks around $500 \mathrm{~nm}$ not present in their excitation spectra, whereas mRFP1-Q66T has single peaks in both cases. The additional peak indicates the presence of an alternative probably immature form of the chromophore ${ }^{6}$.

All mutant proteins emitted readily detectable fluorescence between $\mathrm{pH} 4.5$ and 7.5 with an additional strong increase in light emission under alkaline conditions. Quantitative assessment of $\mathrm{pH}$ dependence revealed $\mathrm{pKa}$ values of 7.5 to 7.9 (Table 1). Despite these rather high $\mathrm{pKa}$ values, these proteins seemed to work well under the physiological conditions tested, demonstrating their applicability as reporters in vivo (Fig. 1c). In fact, the new mRFP1 mutants are two- to threefold brighter than mRFP1 (Fig. 1c and Table 1), with mRFP1-Q66T reaching $47 \%$ of the brightness of DsRED, a substantial improvement compared with DsRED-Monomer, a commercially available monomeric DsRED mutant ( $8 \%$ brightness of DsRED). Seminative protein gel electrophoresis confirmed that the introduced chromophore mutations did not alter the monomeric state of the proteins (Fig. 1d).

DsRED reached half-maximum fluorescence in approximately $10 \mathrm{~h}$ (Table 1), which is in accordance with published data ${ }^{4,5}$. In contrast, mRFP1 as well as mRFP1-Q66C, mRFP1-Q66S and mRFP1-Q66T showed 11-50 times faster maturation. The $t_{1 / 2}$ values of 12 and $11 \mathrm{~min}$, respectively, are comparable to other fast maturing DsRED mutants and superior to other monomeric red fluorescent proteins such as $\mathrm{mKO}$ and DsRED-Monomer ${ }^{7}$ (Table 1). We measured maturation rates at $37{ }^{\circ} \mathrm{C}$ and found that in contrast to other red fluorescent proteins, folding and chromophore formation of the mRFP1 variants is not compromised at elevated temperatures.

To assess photostability, we expressed the mRFP1 variants in Escherichia coli and assayed photobleaching in living cells using confocal laser scanning microscopy. Stability of the most stable mutant protein, mRFP1-Q66T, was comparable to that of DsRED (Fig. 1e). The original mRFP1 protein displayed a 30-fold lower photostability, when compared with DsRED 5 . Therefore, the Q66T mutation not only improves the brightness, but also mediates a higher photostability under these conditions. Bleaching of mRFP1-Q66C and mRFP1-Q66S, however, was faster than that of DsRED or mRFP1-Q66T (Fig. 1e). Photobleaching experiments

\footnotetext{
${ }^{1}$ Max Planck Institute for Plant Breeding Research, Department of Plant Developmental Biology, Carl von Linne Weg 10, D-50829 Cologne, Germany. ${ }^{2}$ University of Cologne, Department of Botany III, Gyrhofstr. 15, D-50931 Cologne, Germany. Correspondence should be addressed to G.J. (jach@mpiz-koeln.mpg.de) or J.F.U. (joachim.uhrig@uni-koeln.de).

RECEIVED 16 MARCH; ACCEPTED 16 JUNE; PUBLISHED ONLINE 21 JULY 2006; DOI:10.1038/NMETH901
} 


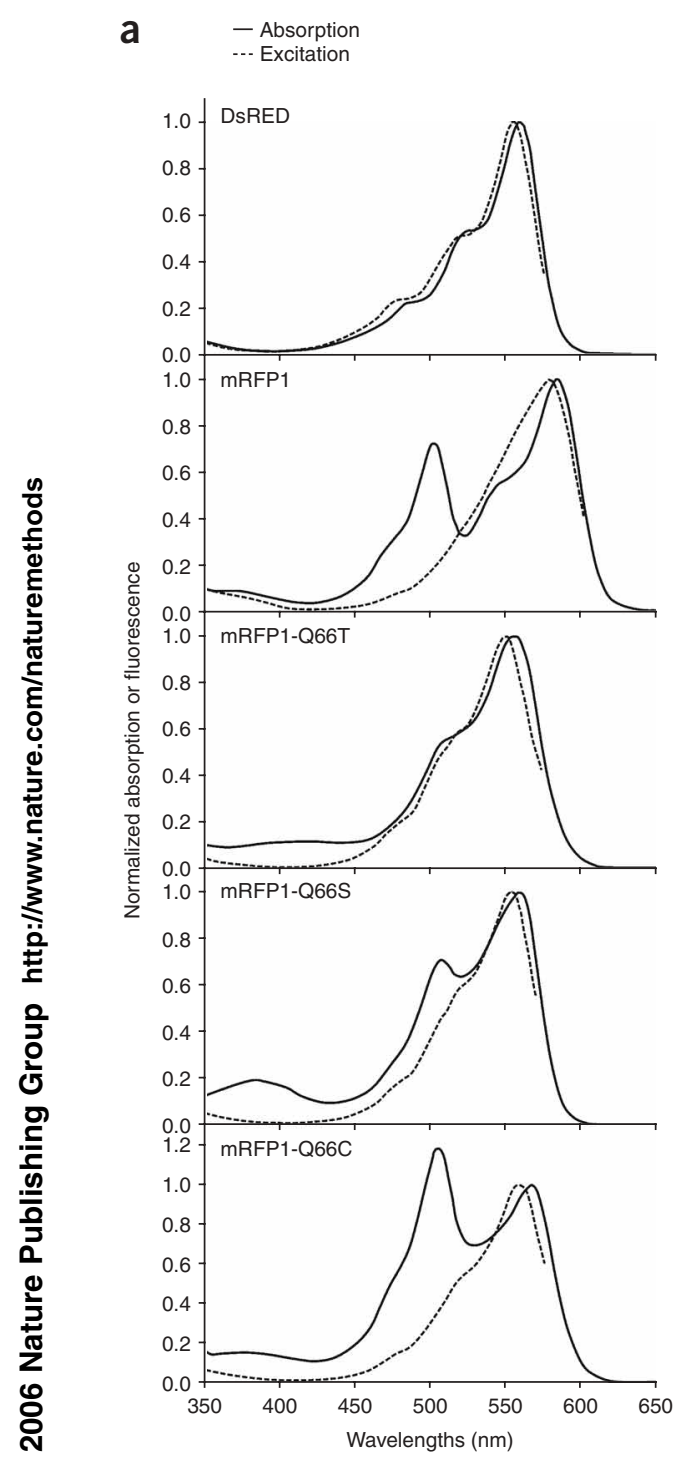

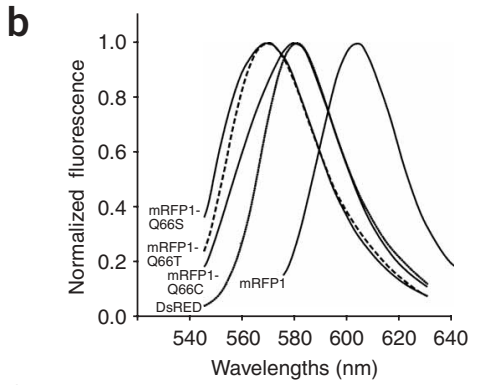

C
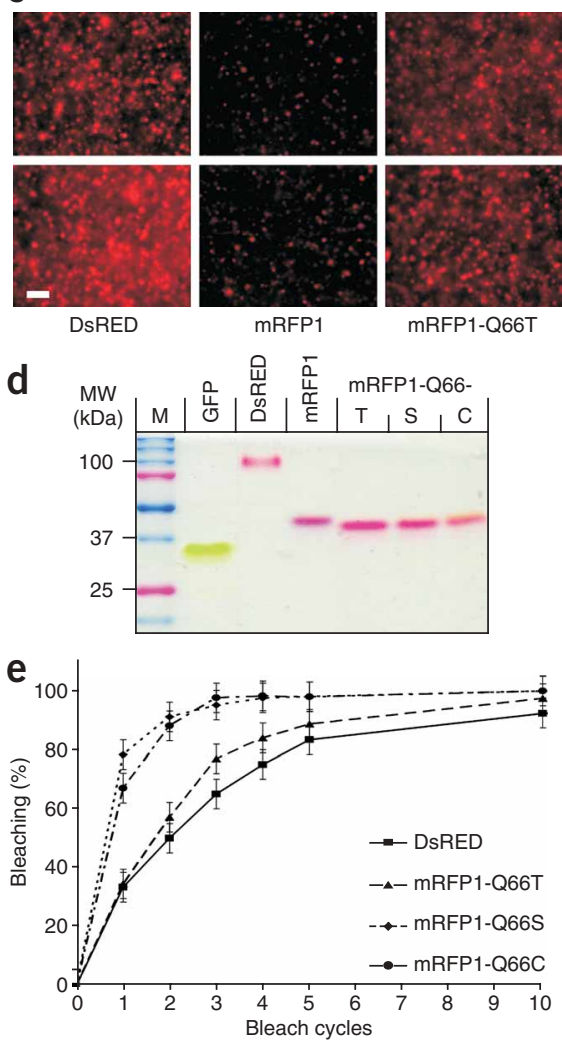

Figure 1 | Spectral properties of new mRFP1 mutants. (a) Absorption and excitation spectra of DsRED, mRFP1, mRFP1-Q66C and mRFP1. Spectra were normalized to the peaks corresponding to the individual excitation maxima. For mRFP1 light emission at $620 \mathrm{~nm}$ was recorded. Emission wavelength for mRFP1-Q66S, mRFP1-066T, mRFP1-066C and wild-type DsRED was $600 \mathrm{~nm}$.

(b) Emission spectra of wild-type DsRED, mRFP1, mRFP1-Q66C, mRFP1-Q66S and mRFP1-Q66T. Measurements were done using excitation wavelengths of $550 \mathrm{~nm}$ (mRFP1) and $520 \mathrm{~nm}$ (mRFP1-Q66C, mRFP1-Q66S, mRFP1-Q66T, DsRED). All curves are normalized to the individual excitation peak values. (c) Transient expression of the fast-maturing DsRED.T3 (ref. 4), mRFP1 and mRFP1-Q66T in tobacco protoplasts. Protoplast suspensions of similar density were analyzed by fluorescence microscopy at low magnification. Two independent experiments are shown. Exposure time, $4 \mathrm{~s}$. Scale bar, $50 \mu \mathrm{m}$. (d) Affinity-purified samples of mRFP1, mRFP1-Q66C, mRFP1-Q66S and $\mathrm{mRFP1}$-Q66T were separated by semi-native SDS-PAGE. GFP and DsRED served as references for monomeric and tetrameric proteins. $M$, marker. (e) Photobleaching of DsRED, mRFP1-066T, mRFP1-Q66S and mRFP1-066C expressed in bacteria. Bacterial cells were bleached with a $514 \mathrm{~nm}$ laser for the indicated number of cycles (duration per cycle, $1.15 \mathrm{~s}$ ). Percent bleaching was calculated from the relative intensity of red fluorescence before and after bleaching. All measurements were repeated at least three times. Error bars, s.d. $(n=3)$.

Guided by structural similarities of DsRED and GFP, we chose two positions in loop regions (amino acids 154 and 168) to split the protein. We fused the respective fragments of mRFP-Q66T to the immunologically detectable myc or HA epitopes, respectively, to serve as flexible in vitro, using purified protein in microdroplets, confirmed the increased photostability of mRFP1-Q66T compared with mRFP1, mRFP1-Q66S and mRFP1-Q66C, respectively (data not shown).

During the course of this study reports of improved mRFP1 variants were published ${ }^{8}$. Notably, some of these mutants also carried the fluorophore mutation Q66T, but always in concert with additional mutations. The data presented in this study emphasize the amino acid at position 66 as being the major determinant of the spectral properties, maturation and photostability. Our in vitro and in vivo data demonstrate that mRFP1-Q66T represents an improved, useful and readily detectable reporter protein with fluorescence levels comparable to that of the brightest monomeric DsRED variants presently available. mRFP1-Q66T represents a good compromise between enhanced brightness and fast maturation because the additional mutations present in mOrange ${ }^{8}$, while increasing the brightness twofold, seem to negatively affect the maturation time (Table 1).

Availability of the enhanced variant mRFP-Q66T provided the basis for the implementation of an RFP-based BiFC system. linkers facilitating the reconstitution of fluorescent protein under nonfavorable steric conditions (see Supplementary Methods online).

Humanized Renilla GFP (hrGFP) forms stable homodimers ${ }^{9}$ and was used in this study as both a fluorescent tag to monitor expression of mRFP1-Q66T fragments and a model protein-protein interaction. We inserted fusions of hrGFP with the $\mathrm{N}$ - and C-terminal mRFP-Q66T fragments (hrGFP-N, hrGFP-C, hrGFP-N2, hrGFP-C2; Fig. 2a) into plant expression vectors containing the CaMV35S promoter (see Supplementary Methods).

Coexpression in tobacco protoplasts of hrGFP-N2 with hrGFP-C or hrGFP-C2, respectively, produced strong red fluorescence, indicating functional BiFC (Fig. 2b). The combination hrGFP-N and hrGFP-C, corresponding structurally to fragments used in the split CFP, BFP, GFP and YFP systems, did not yield any detectable BiFC signal. Transient expression of either construct alone or in combination with mRFP-N or mRFP-C, respectively, served as a control. This did not result in RFP fluorescence, proving that these mRFP1-Q66T fragments do not self-associate (Supplementary Fig. 1 online). The combination of the mRFP1-Q66T fragments 
Table 1 | Quantum yield, extinction coefficient and relative brightness of the new mRFP1 variants

\begin{tabular}{|c|c|c|c|c|c|c|c|}
\hline Protein & $\begin{array}{c}\text { Excitation } \\
\text { maximum }(\mathrm{nm})\end{array}$ & $\begin{array}{c}\text { Emission } \\
\text { maximum }(\mathrm{nm})\end{array}$ & $\begin{array}{c}\text { Extinction } \\
\text { coefficient }\left(\mathrm{M}^{-1} \mathrm{~cm}^{-1}\right)\end{array}$ & $\begin{array}{c}\text { Fluorescence } \\
\text { quantum yield }\end{array}$ & $\begin{array}{c}\text { Maturation } \\
\text { rate }^{\mathrm{a}} t_{1 / 2}(\mathrm{~h})\end{array}$ & $\mathrm{pKa}$ & $\begin{array}{c}\text { Relative } \\
\text { brightness }^{\mathrm{b}}\end{array}$ \\
\hline DsRED & 554 & 581 & $51,500^{c}$ & 0.80 & 9.9 & 4.6 & 1 \\
\hline DsRED-Monomer ${ }^{\mathrm{d}}$ & 556 & 586 & 35,000 & 0.10 & ND & 4.5 & 0.08 \\
\hline mRFP1 & 580 & 604 & 41,800 & 0.15 & 0.2 & 4.5 & 0.15 \\
\hline mRFP1-066C & 559 & 580 & 31,800 & 0.33 & 0.18 & 7.9 & 0.26 \\
\hline mRFP1-Q66S & 555 & 569 & 32,900 & 0.35 & 0.9 & 7.9 & 0.28 \\
\hline mRFP1-Q66T & 549 & 570 & 38,100 & 0.43 & 0.6 & 7.5 & 0.41 \\
\hline mBanana $^{d}$ & 540 & 553 & 6,000 & 0.70 & 1.0 & 6.7 & 0.07 \\
\hline m0range ${ }^{d}$ & 548 & 562 & 71,000 & 0.69 & 2.5 & 6.5 & 0.83 \\
\hline $\mathrm{mKO} 0^{\mathrm{d}}$ & 548 & 559 & 51,600 & 0.60 & 4.5 & 5 & 0.75 \\
\hline mTangerine ${ }^{d}$ & 568 & 585 & 38,000 & 0.30 & ND & 5.7 & 0.19 \\
\hline mCherry $^{d}$ & 587 & 610 & 72,000 & 0.22 & 0.25 & $<4.5$ & 0.38 \\
\hline
\end{tabular}

aMaturation rate at $37^{\circ} \mathrm{C}$; ND, not determined. bBrightness values (extinction coefficient $\times$ quantum yield) in relation to wild-type DsRED protein. ${ }^{\mathrm{C}}$ Published values for the extinction coefficient of DsRED vary considerably $(52,000-75,000)$. Our measured value corresponds to the majority of published values and was used for all calculations (thus representing the minimum brightness to be expected from wild-type DsRED). ${ }^{\text {All }}$ data are from reference 7 , except relative brightness values.

N2 and C2 (Fig. 2a) showed the highest signal-to-noise ratio, and we therefore chose it for further experiments.

Aequorea GFP possesses a residual tendency to dimerize, which is abolished by the point mutation F223R (ref. 10). These proteins can therefore serve as models for weakly or noninteracting proteins. We fused the GFP variant Emerald and the monomeric mEmerald-F223R to the split mRFP-Q66T constructs. Transient expression in protoplasts (Fig. $2 \mathbf{c}, \mathbf{d}$ ) revealed that the RFP-based BiFC system is suitable to detect even the weak Emerald homodimerization (E-N2 and E-C2), whereas noninteracting fusion partners (mE-N2 and mE-C2) do not cause red fluorescence (Supplementary Fig. 1). The ratio of red to green fluorescence was 15-20-fold higher when comparing interacting proteins with controls (Fig. 2d). Intensity of positive signals, on average, reached approximately $30 \%$ of the detected GFP emission. These findings are in good accordance with data from other split fluorescent protein systems.

The readily detectable red fluorescence, using dimeric hrGFP as a model binding protein and fluorescent tag simultaneously, demonstrates the suitability of mRFP1-Q66T fragments for BiFC. The reconstituted fluorescence intensities were comparable in strength with split YFP-based BiFC. This allows analysis by confocal laser scanning microscopy or conventional fluorescence microscopes (Fig. 2c,e). Moreover, our results with the weakly dimerizing GFP variant Emerald indicate that this RFP-based BiFC system is sensitive enough to detect weak protein interactions (Fig. 2c).

Fluorescent proteins commonly form head-to-tail dimers, which, depending on the protein, can interact to form tetramers ${ }^{11}$. Assuming that hrGFP also forms head-to-tail dimers, the different RFP fragments should stick out toward the opposing ends of the complex. Nevertheless, we detected red fluorescence, indicating that the system's flexibility is sufficient to allow BiFC even under unfavorable steric conditions.

To determine the applicability of the RFP-based BiFC to study protein interactions in living organisms, we investigated dimerization of plant transcription factors expressed transiently in leaf epidermal cells. In accordance with published data ${ }^{12}$ interaction between MYB and BHLH transcription factor family members (CPC and GL3, respectively; see Supplementary Methods and
Supplementary Fig. 2 online) could be detected by a red fluorescent signal in the nucleus (Fig. 2e,f). As a negative control, we used CPC containing a single amino acid substitution (R63D) in the MYB domain, shown previously to specifically disrupt the interaction $^{12}$. Transformed cells exhibited no detectable red fluorescence (Fig. 2f), confirming the specificity of the BiFC result. In vivo, homo- and heterodimerization of the plant homeodomain transcription factors BP/KNAT1 and BLH7 was also detected (Supplementary Fig. 2).

A potential problem of current GFP variant-based BiFC systems is their temperature dependence. Low temperatures are favorable and elevated temperatures can be problematic. In this study we performed red BiFC experiments in plant cells at moderate temperatures $\left(26-28^{\circ} \mathrm{C}\right)$. Whether our red BiFC system is also useful at higher temperatures remains to be tested.

Protein aggregation is another potential problem when investigating proteins fused to mRFP1. In some cases this has been reported to interfere with proper intracellular localization of fusion constructs or to compromise cellular structures such as the actin or tubulin cytoskeleton ${ }^{13,14}$. We observed no mislocalization or aggregation in this study. Moreover, our results with plant transcription factors demonstrate that mRFP-based BiFC does not interfere with nuclear targeting. Future experiments will determine whether this system is suitable to analyze proteins associated with the cytoskeleton or targeted to specific membranous compartments.

Association of GFP or YFP fragments in BiFC is supposed to be irreversible. Therefore, a possible drawback of this system is that the dimerization of the fused interacting proteins may also be irreversible. This could influence the cell's physiological state and impede the investigation of dynamic protein interactions. It remains to be investigated whether mRFP1-based BiFC offers an improvement in this respect.

The development of an mRFP-based BiFC system represents an important contribution to broaden the potential of noninvasive fluorescence-based analyses of protein interactions in vivo. Monitoring multiple protein interactions simultaneously in living cells with resolution of the subcellular localization will be of vital importance for further understanding of the complex networks underlying the organization of living cells. 
a

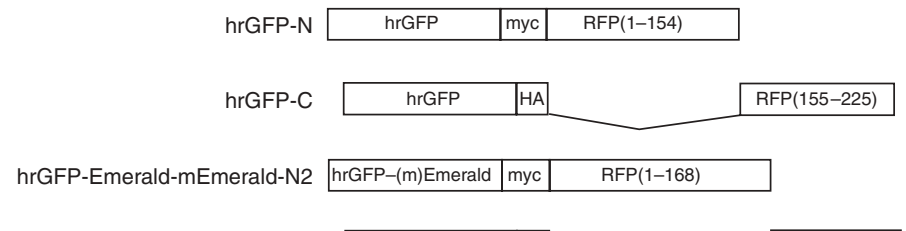

b

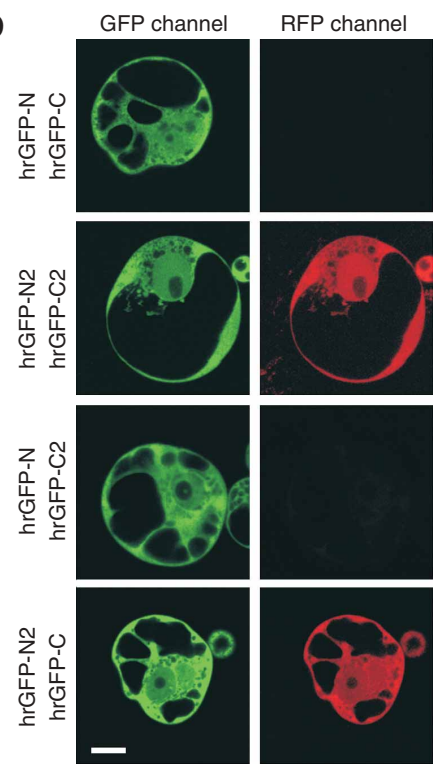

hrGFP-Emerald-mEmerald-C

\begin{tabular}{|l|l|}
\hline hrGFP-(m)Emerald & $\mathrm{HA}$ \\
\hline
\end{tabular}

C

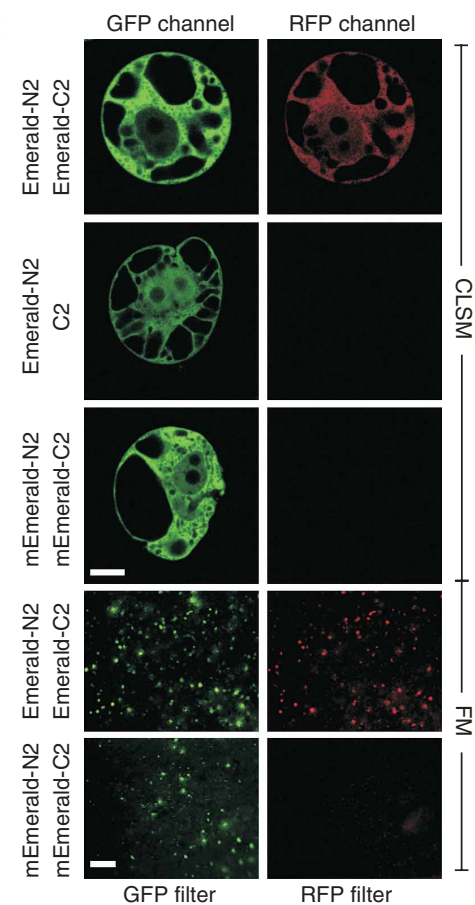

d

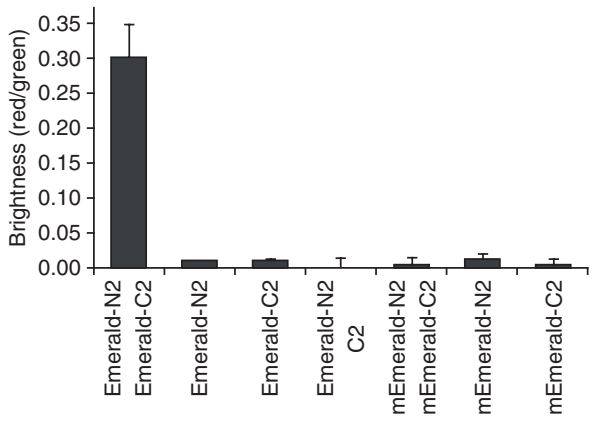

e

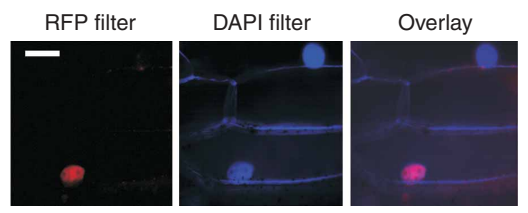

f

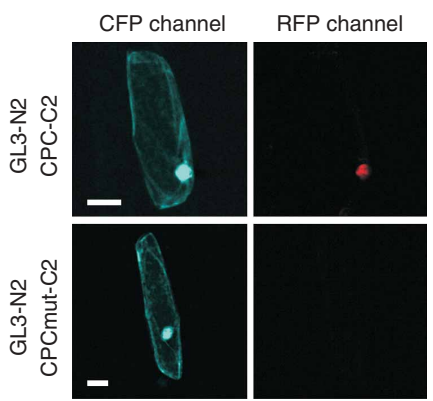

Figure 2 | Bimolecular fluorescence complementation of mRFP1-Q66T fragments. (a) Schematic view of the fusion protein constructs used in this study. $(\mathbf{b}, \mathbf{c})$ Transient gene expression studies in tobacco BY2 protoplasts. Cells transfected with the indicated constructs were incubated overnight at $26{ }^{\circ} \mathrm{C}$ and analyzed by confocal laser scanning microcopy (CLSM) or conventional fluorescence microscopy (FM) at low magnification ( $5 \times$ ). (d) Quantitative analysis of the GFP and RFP signal strength obtained upon transient expression of various construct combinations in tobacco BY2 suspension culture cells. (e,f) FM and CSLM analyses of oligomerization and localization of either GL3-N2/CPC-C2 (e,f) or GL3-N2/CPCmut-C2 (f) proteins coexpressed in onion epidermal cells. In e positions of nuclei are visualized by DAPI staining. N, myc-mRFP1-Q66T-N; N2, myc-mRFP1-Q66T-N2; C, HA-mRFP1-Q66T-C; C2, HA-mRFP1-066T-C2; E, Emerald; mE, mEmerald (monomeric); GL3, GLABRA3, an A. thaliana BHLH protein; CPC, CAPRICE, an A. thaliana MYB protein; CPCmut, mutant CPC with the amino acid exchange R63D. Scale bars: $\mathbf{b}, 10 \mu \mathrm{m} ; \mathbf{c}, 10 \mu \mathrm{m}$ (CLSM) and $500 \mu \mathrm{m}(\mathrm{FM}) ; \mathbf{e}, \mathbf{f}, 50 \mu \mathrm{m}$.

Note: Supplementary information is available on the Nature Methods website.

\section{ACKNOWLEDGMENTS}

We thank G. Coupland and M. Hülskamp for support, and M. Mutondo for critically reading the manuscript. The work was supported by grants from the Max Planck Society.

\section{COMPETING INTERESTS STATEMENT}

The authors declare that they have no competing financial interests.

\section{Published online at http://www.nature.com/naturemethods/} Reprints and permissions information is available online at http://npg.nature.com/reprintsandpermissions/

1. Hu, C.D., Chinenov, Y. \& Kerppola, T.K. Mol. Cell 9, 789-798 (2002).

2. Hu, C.D. \& Kerppola, T.K. Nat. Biotechnol. 21, 539-545 (2003).
3. Knop, M., Barr, F., Riedel, C.G., Heckel, T. \& Reichel, C. Biotechniques 33, 592-602 (2002).

4. Bevis, B.J. \& Glick, B.S. Nat. Biotechnol. 20, 83-87 (2002).

5. Campbell, R.E. et al. Proc. Natl. Acad. Sci. USA 99, 7877-7882 (2002).

6. Verkhusha, V.V., Chudakov, D.M., Gurskaya, N.G., Lukyanov, S. \& Lukyanov, K.A. Chem. Biol. 11, 845-854 (2004).

7. Shaner, N.C., Steinbach, P.A. \& Tsien, R.Y. Nat. Methods 2, 905-909 (2005).

8. Shaner, N.C. et al. Nat. Biotechnol. 22, 1567-1572 (2004).

9. Felts, K. et al. Strategies 13, 85-87 (2000).

10. Zacharias, D.A., Violin, J.D., Newton, A.C. \& Tsien, R.Y. Science 296, 913-916 (2002).

11. Yarbrough, D., Wachter, R.M., Kallio, K., Matz, M.V. \& Remington, S.J. Proc. Natl. Acad. Sci. USA 98, 462-467 (2001).

12. Zimmermann, I.M., Heim, M.A., Weisshaar, B. \& Uhrig, J.F. Plant J. 40, 22-34 (2004).

13. Verkhusha, V.V. \& Sorkin, A. Chem. Biol. 12, 279-285 (2005).

14. Hirrlinger, P.G. et al. Mol. Cell. Neurosci. 30, 291-303 (2005). 